\title{
The Effect of Aerobic Exercises CASE on the Ocular Parameters and STUDY the Psychic State of Glaucoma Patients
}

\author{
J. Lipkova1, T. Gregor ${ }^{1}$, O. Kyselovicova ${ }^{1}$, M. Skodova ${ }^{2}$ \\ ${ }^{1}$ Faculty of Physical Education and Sports, Comenius University Bratislava \\ ${ }^{2}$ Health Center Fedinova Bratislava - Department of Ophtalmology
}

Correspondence to: Janka Lipkova, MD

Faculty of Physical Education and Sports

Comenius University

Bratislava

Slovakia

Key words:

aerobic exercise, glaucoma, psychical state, intraocular pressure, visual field

Activitas Nervosa Superior 2008;50:1-2, 15-17

Abstract Aim of the study was to assess the role of the physical activity in therapy of glaucoma - to find out the effect of aerobic exercise on visual field, intraocular pressure and the psychical state.

The study group consisted of fifteen glaucoma patients - women, mean age 49 years. Eight of them were enrolled in an aerobic exercise program of duration of 3 months. Seven patients - women, matched in age without any organized physical activity serve as control.

The Octopus glaucoma program was used for quantification of the visual field defects. Intraocular pressure was measured by using Goldmann's and Schiotz's tonometer.

EPI (Eysenck Personality Inventory) and STAI (State and Trait Anxiety inventory) were used in evaluating the psychical state.

The values of IOP before and after single exercise session were in normal range.

There were no significant differences between the input and output values of the parameters of visual field. Intraocular pressure in course of the exercise program period was stable, in normal ranges in experimental and in control group as well.

The values of examined persons were in the center of the scale stability-lability and extraversion-introversion. The values of anxiety (as a trait) were in norm, and they did not changed in course the period. The anxiety (as a state) significantly decreased $(\mathrm{p}<0,05)$ in experimental group.

The glaucoma ocular parameters were kept in normal range during the exercise program period. The exercise did not cause the worsening of the ocular parameters after the single (individual) session. A positive effect of exercise program on the anxiety was ascertained. 
$\mathrm{P}$ hysical activity is a very important part of the health lifestyle and a preventive means of the civilization illnesses. However, it may be used as an effective supplementary means in complex treatment of many diseases as well.

Glaucoma

- is a group of illnesses that are often (but not always) accompanied by an increased intraocular pressure (IOP) caused by worse intraocular liquid outflow

- represents an asymptomatic, progressive optic neuropathy characterized by acquired loss of retinal ganglion cells and atrophy of optic nerve. The loss of nerve fibers causes a permanently decreased visual field.

Besides the traditional therapy of glaucoma with topical drugs, the alternative practices, such as megavitamin and herbal therapy, meditation, acupuncture, homeopathic remedies and exercise are used by glaucoma patients. Aerobic exercise is known to lower intraocular pressure (IOP) which protects retinal ganglion cells. Based on a review of the literature no available topical drugs provide neuroprotection or have an effect on ocular blood flow to the retinal cells and so the aerobic exercise may be used as an effective supplementary means in complex treatment of glaucoma.

Psychical stress is an important psychosomatic parameter and a risk factor in pathogenesis of many illnesses. It is known that the hard psychical stress may increase the IOP (Flammer 2003). The unfavorable outlook to the future, the menace of the gradual worsening (even lost) of the sight may alter the patients psychical state. Physical activity may play an important role in reducing the stress and improving the psychical state and the parameters of the glaucoma (IOP and visual field) as well.

Therefore we supposed besides the beneficial effect of aerobic exercises on the parameters of glaucoma the improvement of the psychical state as well.

\section{OBJECTIVES}

of the study was to assess the role of the physical activity in therapy of glaucoma - to find out the effect of aerobic exercise on visual field, intraocular pressure and the psychical state.

TASKS:

1. To evaluate the short-term effect of the aerobic exercises on IOP - to find out the differences between the levels of IOP before and after exercise session.

2. To compare the input and output levels of parameters of visual field (mean sensitivity and mean difference).
3. To find out the trend of the IOP in the exercise program period.

4. To find out the changes in psychical state due to 3month training

\section{SUBJECTS}

\begin{tabular}{|l|l|l|}
\hline & Target group (A) & Control group (B) \\
\hline Subjects & 8 female patients & 7 female patients \\
\hline Dg & $\begin{array}{l}\text { open angle } \\
\text { glaucoma }\end{array}$ & open angle glaucoma \\
\hline Age (years) & $49,4 \pm 2,2$ & $48,9 \pm 2,3$ \\
\hline $\begin{array}{l}\text { Exercise program } \\
\text { Furation of the } \\
\text { session } \\
\text { Period }\end{array}$ & $\begin{array}{l}3 \text {-times a week } \\
\text { Medication }\end{array}$ & $\begin{array}{l}\text { any organized } \\
\text { physical activity }\end{array}$ \\
\hline
\end{tabular}

\section{METHODS:}

\section{Exercise program:}

The classes combined both the aerobic and strength phase as well (Body Form Aerobics 2x and Fit ball Aerobics 1x per week). During each session the standard structure was kept: Warm - up. 10 to 15 minutes low impact aerobics followed by pre - stretching. Main aerobics part: 20 - to 30 minutes of aerobics movements done continuously or in intervals within aerobic training zones of individuals (50 - 85\% HRmax.). Cool down: 5 minutes of slow relaxation, mostly with stretches for the particular muscle groups.

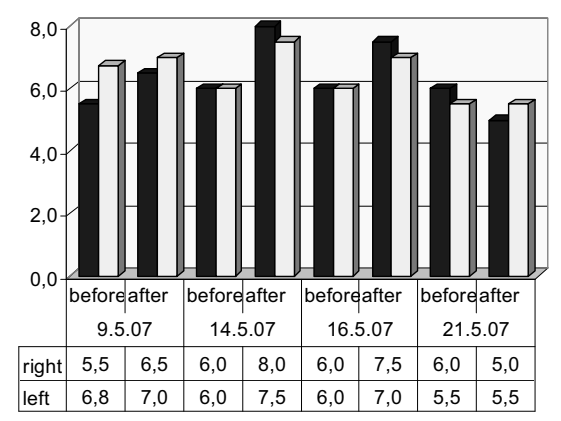

Fig. 1 Intraocular pressure before and after the exercise

Perimetry:

Quantification of the defects in visual field was evaluated by using the Octopus glaucoma program. Intraocular 
pressure was measured by using Schiotz's tonometer (in the gymnasium) and by Goldmann's tonometer (in the Health center - department of ophthalmology).

Psychological state:

In evaluating the psychological state the EPI (Eysenck personality inventory) and STAI (State and trait anxiety inventory) were used .

\section{RESULTS:}

Analysis of data collected showed

1. - significant increase of intraocular pressure after single exercise was found out only in one exercise session (fig. 1) 2. - any significant changes in parameters of visual field neither in mean defect nor in mean sensitivity (fig.2, 3)

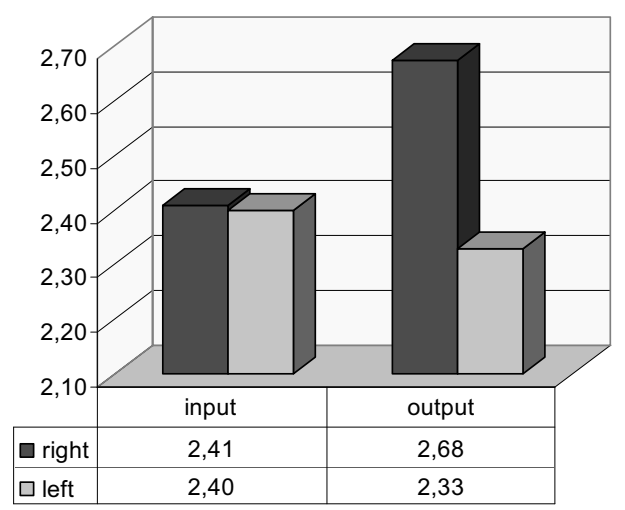

Fig. 2 Visual field difference

3. - trend of the intraocular pressure values in course the exercise program period was stable in exercise and control group as well (fig.4, 5)

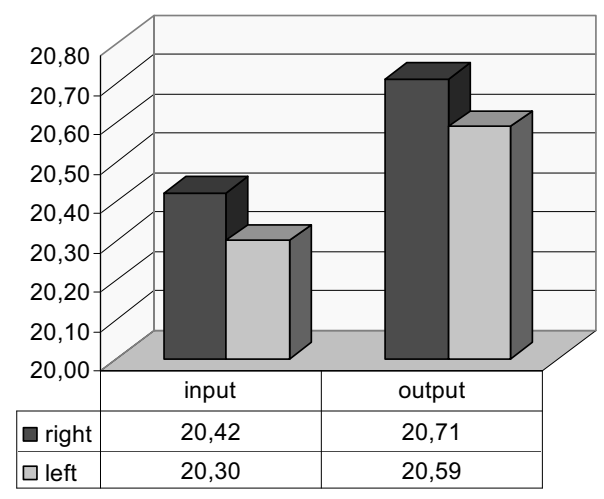

Fig. 3 Visual field - mean sensitivity

4. - Analysis of EPI showed, that the personality of probands were balanced. They were nor extroverts, neither introverts. The values of anxiety (as a trait) were in norm, and they did not changed in course the period. Level of anxiety (as a state) decreased after the 3 month of exercise training.

\section{CONCLUSION:}

The short-time effect of aerobic exercise on intraocular pressure was not proved.

Parameters of visual field have not changed after the 3month period of aerobic training.

Intraocular pressure in course of the exercise program period was stable.

The values of parameters of anxiety decreased after the exercise training program.

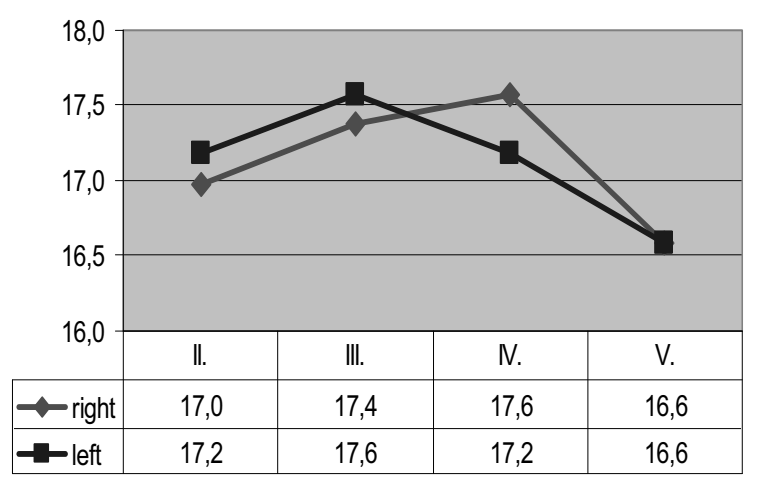

Fig. 4 Intraocular pressure - target group

We have not succeeded in proving unambiguously a positive effect of aerobic exercises on the ocular parameters in glaucoma patients. On the other hand the exercise did not cause the worsening of the state neither after the single (individual) session nor in the course of the exercise program

period.

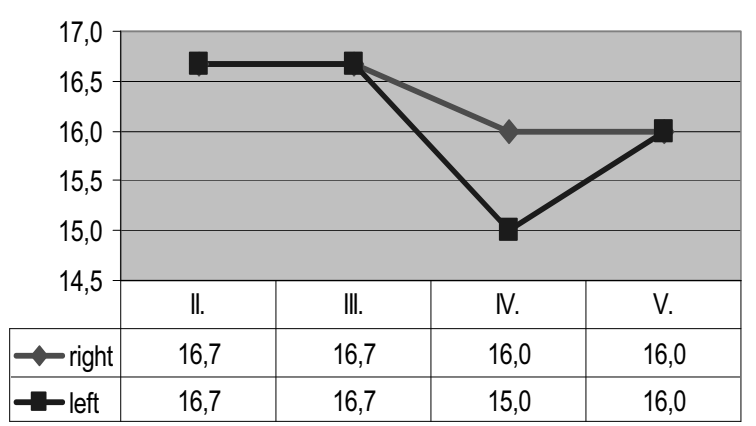

Fig. 5 Intraocular pressure - control group

The values of ocular parameters were in norm and they have not changed neither in experimental nor in control group. Nevertheless, also the positive effects of exercise on psychical state should be taken in account.

\section{REFERENCES}

Flammer J (2003). Glaukom. Praha: Triton 